\title{
OPTIMIZATION OF EIGENFREQUENCIES OF PSEUDO 3D COMPOSITE
}

\author{
P. P. Prochazka
}

Department of Structural Mechanics, Czech Technical University in Prague, Civil Engineering, Prague, Czech Republic (petrp@fsv.cvut.cz)

\begin{abstract}
In this paper an optimization of laminated arches is solved under condition that generally anisotropic layers are considered and the design parameters of optimization are eigenparameters (intrinsic fields), either eigenstrains or eigenstresses. Mathematical apparatus is to be discussed and the formulas needed for programming on computers will be derived. The focus here is concentrated on problem of the response of harmonic load, which can be simulated by developing time coordinate into the Fourier series. The solution on separated coordinates of position is formulated for simply supported or clamped layered arch in cylindrical coordinates. The semi-analytic solution is used for simply supported arch in the position coordinates. The conditions being valid for clamped edges are taken from the well known Lechnitski's book on Theory of plates. The procedure used in the book enables us to solve the simply supported arch and by virtue of unit impulses of a slope at the supports to derive the solution for fixed ends. The boundary conditions are fulfilled by selections of sine or cosine series applied to different directions of displacements and eigenparameters. Then, the static case is described in each lamina separately and the overall relations are provided with fulfillment of interfacial conditions being valid on the interfaces of the adjacent laminas. Since the influence of inertia forces appear to be quite simple, the problem is focused on the separated static case (in the position coordinates). The formulation leads to the solution of a simultaneous system of ordinary differential equations, which are defined in one generic lamina. In our case harmonic load is discussed, as eigenfrequencies are of the main interest to us. The pseudo 3D formulation is based on generalized plain strain, so that also axial direction can be taken into account in the optimization. Examples are presented starting with twodimensional case, which is based on plain strain formulation, simplifying the general case.
\end{abstract}

Keywords: Optimization of laminated arches, Eigenparameters, Eigenfrequencies, Stationary point of Hamiltonian.

\section{INTRODUCTION}

There is couple of papers solving vibration of arches, particularly the effect of sudden load impact. A coupled formulation based on the semi-analytical finite element technique is developed for composite arches conveying fluid is presented in paper [1]. 
The structural finite element formulation is from [2] while the fluid part is modeled by the characteristic wave equation. The fluid part is modeled using a velocity potential formulation and the dynamic pressure acting on the walls is derived from Bernoulli's equation. Impermeability and dynamic condition are imposed on the fluid-structure interface. The finite element equations for the composite arches conveying fluid are validated using available results. A detailed parametric study is carried out for various boundary conditions as well as for different length-to-radius and radius-to-thickness ratios.

Static and dynamic characteristics of thick composite laminates exposed to hydrothermal environment are studied in [3] using a realistic higher-order theory developed recently. The formulation accounts for the nonlinear variation of the in-plane and transverse displacements through the thickness, and abrupt discontinuity in slope of the in-plane displacements at any interface.

Paper [4] presents a curved finite element model for the three-dimensional nonlinear analysis of elastic arches. The model includes higher-order curvatures which make the order of the bending strains consistent with that of the membrane strains, and the same low-order polynomials are used for all the displacements. No approximations are made for the twist rotations.

Arches are often connected to other members, which influence the structural behavior of the arch. These members induce restraining actions during flexural-torsional buckling which restrict the buckled shapes of the arch and may significantly influence its buckling response. The paper [5] uses an energy method to study the elastic flexural-torsional buckling of continuously restrained arches of doubly symmetric open thin-walled cross-section in uniform bending and in uniform axial compression.

An elastic buckling theory is developed in [6] for thin-walled arches. Using the principle of minimum total potential energy derives the governing differential equations. An explicit and clear approximation of the curvature effect is made in the derivation process. Closed form solutions are obtained for arches subjected to equal and opposite end moments (uniform bending) and to uniformly distributed radial loads (uniform compression).

In this paper general cylindrical thick-walled arch is solved for dynamic and static behavior. The formulation leads to the solution of a simultaneous system of ordinary differential equations, which are defined in one genetic lamina. Generalized plane strain state is considered, which enables to split the solution into two stages: one being connected with plain strain state and the second with axial compression or tension. This conception model is very useful in practice and concurrently simplifies the solution of the stress states in the structures envisaged. Boundary and interfacial conditions provide overall response on external load.

The optimization is a generalization of seeking the stationary point of constrained Lagrangian, [7]. An example of the solution starts with one wave of the load.

In this paper a generalization of the previously mentioned paper is put forward when Lagrangian is substituted by Hamiltonian to get relation between eigenfrequencies and eigenparameters. The basic formulation is found in [8]. 


\section{BASIC ARRANGEMENT}

We start with introducing the polar coordinates $\operatorname{0r} \theta z$, which are derived from Cartesian coordinates $0 x y z$. Components of the displacement vector are considered as: $u=u_{r}, v=u_{\theta}, w=u_{z}$, where $u_{r}$ is the displacement in the radial direction and $u_{\theta}$ is the displacement in the circumferential (hoop) direction and $w=u_{z}$ is the displacement field in the axial direction, being uniformly distributed in the $z$ - direction. The geometry of the entire laminated arch is depicted in Fig. 1.

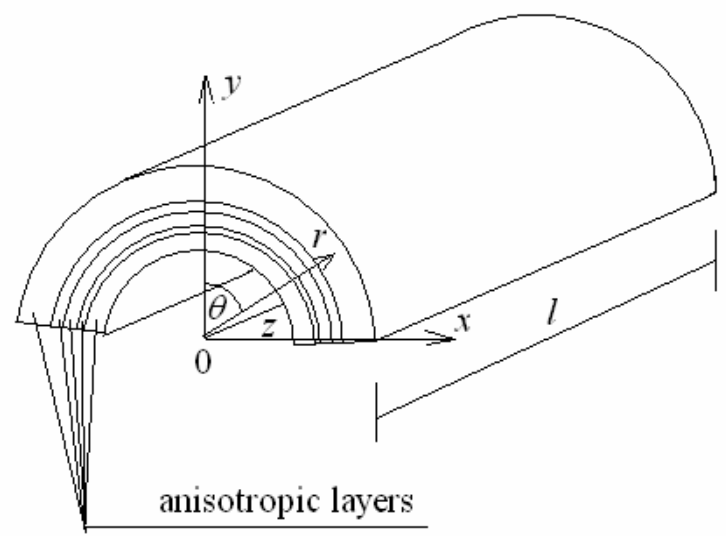

Figure 1. Geometry and coordinate system of the entire cylindrical arch

\section{BASIC ARRANGEMENT}

In this section focus is put on one lamina from laminate cylindrical segment (arch). The cylindrically anisotropic structure is described by Hooke's law in the selected lamina (the number of the lamina is dropped out as the relations are valid for all laminas, so that we can consider the following relations as typical one):

$$
\begin{aligned}
& \text { in vector notation } \sigma_{i}=L_{i j} \varepsilon_{j}+\mu_{i}, L_{i j}=L_{j i}, \\
& \text { or } \boldsymbol{\sigma}=\boldsymbol{L} \boldsymbol{\varepsilon}+\boldsymbol{\mu} \text {, or in tensor notation } \sigma_{i j}=L_{i j k l} \varepsilon_{k l}+\mu_{i j}, \quad i, j, k, l=r, \theta, z
\end{aligned}
$$

where

$$
\boldsymbol{L}=\left[\begin{array}{cccccc}
L_{r r} & L_{r \theta} & L_{r z} & 0 & 0 & 0 \\
L_{r \theta} & L_{\theta \theta} & L_{\theta z} & 0 & 0 & 0 \\
L_{r z} & L_{\theta z} & L_{z z} & 0 & 0 & 0 \\
0 & 0 & 0 & G_{r \theta} & 0 & 0 \\
0 & 0 & 0 & 0 & G_{r z} & 0 \\
0 & 0 & 0 & 0 & 0 & G_{\theta z}
\end{array}\right]
$$

and

$$
\boldsymbol{\sigma}=\left\{\sigma_{r}, \sigma_{\theta}, \sigma_{z}, \sigma_{r \theta}, \sigma_{r z}, \sigma_{\theta z}\right\}^{\mathrm{T}} \quad \boldsymbol{\varepsilon}=\left\{\varepsilon_{r}, \varepsilon_{\theta}, \varepsilon_{z}, \varepsilon_{r \theta}, \varepsilon_{r z}, \varepsilon_{\theta z}\right\}^{\mathrm{T}}
$$


The kinematical equations are written in polar coordinates as:

$$
\begin{gathered}
\varepsilon_{r}=\frac{\partial u}{\partial r}, \quad \varepsilon_{\theta}=\frac{1}{r} \frac{\partial v}{\partial \theta}+\frac{u}{r}, \quad \varepsilon_{\mathrm{z}}=\frac{\partial w}{\partial z} \\
\varepsilon_{r \theta}=\frac{1}{r} \frac{\partial u}{\partial \theta}+\frac{\partial v}{\partial r}-\frac{v}{r}, \quad \varepsilon_{r z}=\frac{\partial w}{\partial r}+\frac{\partial u}{\partial z}, \quad \varepsilon_{\theta z}=\frac{\partial v}{\partial z}+\frac{1}{r} \frac{\partial w}{\partial \theta}
\end{gathered}
$$

Neglecting the volume weight three equations of equilibrium provide relations among components of the stress tensor:

$$
\begin{gathered}
\frac{\partial \sigma_{r}}{\partial r}+\frac{\partial \sigma_{r z}}{\partial z}+\frac{1}{r}\left(\frac{\partial \sigma_{r \theta}}{\partial \theta}+\sigma_{r}-\sigma_{\theta}\right)-\rho \frac{\partial^{2} u}{\partial t^{2}}=0, \quad \frac{\partial \sigma_{r \theta}}{\partial r}+\frac{\partial \sigma_{\theta z}}{\partial z}+\frac{1}{r}\left(\frac{\partial \sigma_{\theta}}{\partial \theta}+2 \sigma_{r \theta}\right)-\rho \frac{\partial^{2} v}{\partial t^{2}}=0 \\
\frac{\partial \sigma_{z}}{\partial z}+\frac{\partial \sigma_{r z}}{\partial r}+\frac{1}{r}\left(\sigma_{r z}+\frac{\partial \sigma_{\theta z}}{\partial \theta}\right)-\rho \frac{\partial^{2} w}{\partial t^{2}}=0
\end{gathered}
$$

where $\rho$ is the mass density of the lamina under account, $t$ is time.

We solve now the declared problem on one layer (lamina) without eigenparameters in terms of the semi-analytical method. For this we introduce sine and cosine series and coefficient $\alpha_{\ell}$, which describes the position of a concrete term in the series. Express $\alpha_{\ell}$ as $\alpha_{\ell}=m . \ell$, where positive integer $\ell$ is the number of the wave in the Fourier series and $m=$ $\pi / \beta$. The coefficient $\beta$ denotes the width of end points of the arch.

Expanding three components of displacements into sine and cosine series, introducing the plain strain state condition, and denoting the $\ell$-th term depending only on $r$ and $z$ by $U^{\ell}$ and $V^{\ell}$ yields

$$
\begin{gathered}
u^{\ell}(r, \theta, z, t)=U^{\ell}(r, z) \cos \alpha \theta \exp (\mathrm{i} \omega t), \quad v^{\ell}(r, \theta, t)=V^{\ell}(r, z) \sin \alpha \theta \exp (\mathrm{i} \omega t), \\
w^{\ell}(r, \theta, t)=W^{\ell}(z) \cos \alpha \theta \exp (\mathrm{i} \omega t), \\
\varepsilon_{z}^{\ell}(r, \theta, z, t)=\frac{\partial W^{\ell}(z)}{\partial z} \cos \alpha \theta \exp (\mathrm{i} \omega t)=\bar{\varepsilon}_{z} \cos \alpha \theta \exp (\mathrm{i} \omega t), \quad \text { i.e. } w^{\ell}=\bar{\varepsilon}_{z} z \cos \alpha \theta \exp (\mathrm{i} \omega t)
\end{gathered}
$$

where $U^{\ell}$ and $V^{\ell}$ are unknown functions of $r$ and $z$, and $W^{\ell}$ is a function of $z$ only, which need to be determined from the equations of equilibrium, and $\mathrm{i}$ is the imaginary unit, $\bar{\varepsilon}_{z}$ is a constant to be also determined independently on $\alpha$. In particular, substituting (1) in the stressstrain relations gives stresses which, when substituted into the equations of equilibrium in cylindrical coordinates, provide the equations for evaluation of $U^{\ell}$ and $V^{\ell}$ in each lamina, while $W^{\ell}$ is sought in entire structure, depending only on $z$.

Components of strains, (2), and stresses, (3), are then expressed as (identification $\ell$ of the number in the series is dropped out), 


$$
\begin{array}{r}
\varepsilon_{r}=\frac{\partial U}{\partial r} E \cos \alpha \theta, \quad \varepsilon_{\theta}=\frac{1}{r}(\alpha V+U) E \cos \alpha \theta, \quad \varepsilon_{r \theta}=\left(\frac{-\alpha}{r} U+\frac{\partial V}{\partial r}-\frac{1}{r} V\right) E \sin \alpha \theta \\
\varepsilon_{r z}=\frac{\partial U}{\partial z} E \cos \alpha \theta, \quad \varepsilon_{\theta z}=\left(\frac{\partial V}{\partial z}-\alpha \bar{\varepsilon}_{z} \frac{z}{r}\right) E \sin \alpha \theta, \quad \varepsilon_{z}=\bar{\varepsilon}_{z} E \cos \alpha \theta,
\end{array}
$$

and for simplicity of derivation before and in what follows only one term of Fourier's series will be considered, i.e. $\ell=$ const., $E=\exp (i \omega t)$. Then, the stresses are expressed as

$$
\begin{gathered}
\sigma_{r}=\left[L_{r r} \frac{\partial U}{\partial r}+\frac{L_{r \theta}}{r}(\alpha V+U)+L_{r z}-\bar{\varepsilon}_{z}\right] E \cos \alpha \theta, \quad \sigma_{\theta}=\left[L_{r \theta} \frac{\partial U}{\partial r}+\frac{L_{\theta \theta}}{r}(\alpha V+U)+L_{\theta z} \bar{\varepsilon}_{z}\right] E \cos \alpha \theta \\
\sigma_{z}=\left[L_{r z} \frac{\partial U}{\partial r}+\frac{L_{\theta z}}{r}(\alpha V+U)+L_{z z} \bar{\varepsilon}_{z}\right] E \cos \alpha \theta \\
\sigma_{r \theta}=G_{r \theta}\left[-\frac{(\alpha U+V)}{r}+\frac{\partial V}{\partial r}\right] E \sin \alpha \theta, \quad \sigma_{r z}=G_{r z} \frac{\partial U}{\partial z} E \cos \alpha \theta, \quad \sigma_{\theta z}=G_{\theta z}\left(\frac{\partial V}{\partial z}-\alpha \bar{\varepsilon}_{z} \frac{z}{r}\right) E \sin \alpha \theta
\end{gathered}
$$

Now the particular terms in equation of equilibrium (3) are derived as:

$$
\begin{gathered}
\sigma_{r}-\sigma_{\theta}=\left\{\left(L_{r r}-L_{r \theta}\right) \frac{\partial U}{\partial r}+\left(L_{r \theta}-L_{\theta \theta}\right) \frac{1}{r}(\alpha V+U)+\left(L_{r z}-L_{\theta z}\right) \varepsilon_{z}\right\} E \cos \alpha \theta \\
\frac{\partial \sigma_{r}}{\partial r}=\left\{L_{r r} \frac{\partial^{2} U}{\partial r^{2}}+L_{r \theta}\left[-\frac{1}{r^{2}}(\alpha V+U)+\frac{1}{r} \frac{\partial(\alpha V+U)}{\partial r}\right]\right\} E \cos \alpha \theta \\
\frac{\partial \sigma_{\theta}}{\partial \theta}=-\left[L_{r \theta} \frac{\partial U}{\partial r}+\frac{L_{\theta \theta}}{r}(\alpha V+U)+L_{r z}-\varepsilon_{z}\right] \alpha E \sin \alpha \theta \\
\frac{\partial \sigma_{r \theta}}{\partial \theta}=G_{r \theta}\left[-\frac{1}{r}(\alpha U+V)+\frac{\partial V}{\partial r}\right] \alpha E \cos \alpha \theta \frac{\partial \sigma_{z}}{\partial z}=\left[L_{r z} \frac{\partial^{2} U}{\partial z^{2}}+\frac{L_{\theta z}}{r} \frac{\partial(\alpha V+U)}{\partial z}\right] E \cos \alpha \theta \\
\frac{\partial \sigma_{r \theta}}{\partial r}=G_{r \theta}\left[\frac{1}{r^{2}}(\alpha U+V)-\frac{1}{r} \frac{\partial(\alpha U+V)}{\partial r}+\frac{\partial^{2} V}{\partial r^{2}}\right] E \sin \alpha \theta \\
\frac{\partial \sigma_{r z}}{\partial r}=G_{r z} \frac{\partial^{2} U}{\partial r \partial z} E \cos \alpha \theta \\
\frac{\partial \sigma_{\theta z}}{\partial z}=G_{\theta z}\left(\frac{\partial^{2} V}{\partial z^{2}}-\alpha \bar{\varepsilon}_{z} \frac{1}{r}\right) E \sin \alpha \theta \quad G_{\theta z}\left(\frac{\partial V}{\partial z}-\alpha \varepsilon_{z} \frac{z}{r}\right) \alpha E \cos \alpha \theta \\
\frac{\partial \sigma_{r z}}{\partial z}=G_{r z} \frac{\partial^{2} U}{\partial z^{2}} E \cos \alpha \theta
\end{gathered}
$$

The first equation of equilibrium is listed as:

$$
\begin{aligned}
& 0=L_{r r} \frac{\partial^{2} U}{\partial r^{2}}+L_{r \theta}\left[-\frac{1}{r^{2}}(\alpha V+U)+\frac{1}{r} \frac{\partial(\alpha V+U)}{\partial r}\right]+G_{r z} \frac{\partial^{2} U}{\partial z^{2}}+\rho \omega^{2} U+ \\
& +\frac{1}{r}\left(-\alpha G_{r \theta}\left[-\frac{(\alpha U+V)}{r}+\frac{\partial V}{\partial r}\right]+\left(L_{r r}-L_{r \theta}\right) \frac{\partial U}{\partial r}+\left(L_{r \theta}-L_{\theta \theta}\right) \frac{1}{r}(\alpha V+U)+\left(L_{r z}-L_{\theta z}\right) \bar{\varepsilon}_{z}\right)
\end{aligned}
$$

The second equation reads similarly as: 


$$
\begin{aligned}
& G_{r \theta}\left[\frac{1}{r^{2}}(\alpha U+V)-\frac{1}{r} \frac{\partial(\alpha U+V)}{\partial r}+\frac{\partial^{2} V}{\partial r^{2}}\right]+G_{\theta z}\left(\frac{\partial^{2} V}{\partial z^{2}}-\frac{\alpha-}{r} \bar{\varepsilon}_{z}\right)+ \\
& +\frac{1}{r}\left(-\left[L_{r \theta} \frac{\partial U}{\partial r}+\frac{L_{\theta \theta}}{r}(\alpha V+U)+L_{r z} \bar{\varepsilon}_{z}\right]+2 G_{r \theta}\left[-\frac{(\alpha U+V)}{r}+\frac{\partial V}{\partial r}\right]\right)+\rho \omega^{2} V=0
\end{aligned}
$$

holds:

Now we concentrate our attention on the third equation and try to simplify it. It

$$
\left[L_{r z} \frac{\partial^{2} U}{\partial r \partial z}+\frac{L_{\theta z}}{r} \frac{\partial(\alpha V+U)}{\partial z}\right]+G_{r z} \frac{\partial^{2} U}{\partial r \partial z}+\frac{1}{r} G_{r z}\left[\frac{\partial(U+\alpha V)}{\partial z}-\frac{\alpha^{2} z \bar{\varepsilon}_{z}}{r}\right]+\rho \omega^{2} \bar{\varepsilon}_{z} z=0
$$

The last three equations create a simultaneous system of partial differential equations for unknown $U, V$ and $\bar{\varepsilon}_{z}$. It is necessary to note that while the first two unknowns are valid locally inside each lamina the strain $\bar{\varepsilon}_{z}$ holds for the entire structure.

Closing with the conditions that higher order derivatives of $U$ and $V$ by $z$ are negligible, the terms in (7) and (8) belonging to $G_{r z}$ and $G_{\theta z}$ disappear. Since the material coefficients are independent on the coordinates and $W=\bar{\varepsilon} z$, the integration by $z$ leads us to the relation describing the situation in the axial direction ( $C$ is a function of $r$ only):

$$
\left[L_{r z} \frac{\partial U}{\partial r}+\frac{L_{\theta z}}{r}(\alpha V+U)\right]+G_{r z} \frac{\partial U}{\partial r}+\frac{1}{r} G_{r z}\left[U+\alpha V-\frac{\alpha^{2} z^{2} \bar{\varepsilon}_{z}}{2 r}\right]+\rho \omega^{2} \bar{\varepsilon}_{z} \frac{z^{2}}{2}=C(r)
$$

\section{PLAIN STRAIN}

The above formulated equations are relatively very complicated, they are even nonlinear. They have to be solved in terms similar to folded plates (in our case folded arches) or folded strips. On the other hand some reasonable simplification can provide appropriate solution, which may be considered as precise enough approximation. In any case, if split pure plain strain and the influence of axial direction leads to a rigorous possibility on how to present the solution. Here we concentrate on plain strain part.

First, in what follows the assumption will be adopt: $L_{r r}=L_{\theta \theta}$, i.e. the isotropic medium is presumed inside all laminas, and moreover for such a medium it holds: $L_{r r}-L_{r \theta}=2 G_{r \theta}$.

If the plain strain state is considered, $W=\bar{\varepsilon}=0$. Then the first equation of equilibrium is expressed as:

$$
U^{\prime \prime}+\frac{U^{\prime}}{r}+A_{1} \frac{U}{r^{2}}+A_{2} \frac{V^{\prime}}{r}+A_{3} \frac{V}{r^{2}}=0
$$

where

$$
A_{1}=\frac{-\left(L_{r r}+\alpha^{2} G_{r \theta}\right)}{L_{r r}}+(\bar{r} \omega)^{2}, \quad A_{2}=\frac{\alpha\left(L_{r \theta}+G_{r \theta}\right)}{L_{r r}}, \quad A_{3}=\frac{-\alpha\left(L_{r r}+G_{r \theta}\right)}{L_{r r}}
$$


Under the above condition the second equation of equilibrium is defined as follows:

$$
V^{\prime \prime}+\frac{V^{\prime}}{r}+B_{1} \frac{V}{r^{2}}+B_{2} \frac{U^{\prime}}{r}+B_{3} \frac{U}{r^{2}}=0
$$

where

$$
B_{1}=\frac{-\left(G_{r \theta}+\alpha^{2} L_{r r}\right)}{G_{r \theta}}+(\bar{r} \omega)^{2}, B_{2}=\frac{-\alpha\left(L_{r \theta}+G_{r \theta}\right)}{G_{r \theta}}, B_{3}=\frac{-\alpha\left(L_{r r}+G_{r \theta}\right)}{G_{r \theta}}
$$

and $\bar{r}$ is the average of radii in the trial lamina.

The solution of simultaneous equations (8) and (9) can be done using the substitution: $r=\exp (t)$. Then the above differential equations are solved in standard way to get roots $\lambda$ of characteristic equation of the fourth order to the fundamental solutions: $r^{\lambda_{1}}, r^{\lambda_{2}}, r^{\lambda_{3}}, r^{\lambda_{4}}$. The characteristic equation provides roots:

$$
\lambda^{4}+\left(\mathrm{A}_{1}+\mathrm{B}_{1}-\mathrm{A}_{2} \mathrm{~B}_{2}\right) \lambda^{2}+\mathrm{A}_{1} \mathrm{~B}_{1}-\mathrm{A}_{3} \mathrm{~B}_{3}=0, \lambda^{4}-\mathrm{b} \lambda^{2}+c=0
$$

where $\lambda_{1,2}= \pm \sqrt{\frac{b \pm \sqrt{b^{2}-4 c}}{2}}= \pm(1 \pm \alpha)$.

The fundamental solution for unknown amplitudes of displacements $U$ and $V$ follows as:

$$
\begin{gathered}
U=\left\{-\left(A_{2} \lambda_{1}+A_{3}\right) A r^{\lambda_{1}}+\left(A_{2} \lambda_{1}-A_{3}\right) B r^{-\lambda_{1}}-\right. \\
\left.-\left(A_{2} \lambda_{2}+A_{3}\right) C r^{\lambda_{2}}+\left(A_{2} \lambda_{2}-A_{3}\right) D r^{-\lambda_{2}}\right\} \cos \alpha \theta= \\
=\left\{\left[\left(\lambda_{1}\right)^{2}+B_{1}\right] A r^{\lambda_{1}}+\left[\left(\lambda_{1}\right)^{2}+B_{1}\right] B r^{-\lambda_{1}}+\right. \\
\left.+\left[\left(\lambda_{2}\right)^{2}+B_{1}\right] C r^{\lambda_{2}}+\left[\left(\lambda_{2}\right)^{2}+B_{1}\right] D r^{-\lambda_{2}}\right\} \cos \alpha \theta \\
V=\left\{\left[\left(\lambda_{1}\right)^{2}+A_{1}\right] A r^{\lambda_{1}}+\left[\left(\lambda_{1}\right)^{2}+A_{1}\right] B r^{-\lambda_{1}}+\right. \\
\left.+\left[\left(\lambda_{2}\right)^{2}+A_{1}\right] C r^{\lambda_{2}}+\left[\left(\lambda_{2}\right)^{2}+A_{1}\right] D r^{-\lambda_{2}}\right\} \sin \alpha \theta= \\
=\left\{-\left(B_{2} \lambda_{1}+B_{3}\right) A r^{\lambda_{1}}+\left(B_{2} \lambda_{1}-B_{3}\right) B r^{-\lambda_{1}}-\right. \\
\left.-\left(B_{2} \lambda_{2}+B_{3}\right) C r^{\lambda_{2}}+\left(B_{2} \lambda_{2}-B_{3}\right) D r^{-\lambda_{2}}\right\} \sin \alpha \theta
\end{gathered}
$$

or in matrix notation involving the wave $\ell$ 


$$
\left\{\begin{array}{c}
\frac{U^{\ell}(r)}{\cos \alpha_{\ell} \theta} \\
\frac{V^{\ell}(r)}{\sin \alpha_{\ell} \theta}
\end{array}\right\}=\left[\begin{array}{cccc}
\Sigma^{u}\left(\lambda_{1}^{\ell}\right) & \Sigma^{u}\left(-\lambda_{1}^{\ell}\right) & \Sigma^{u}\left(\lambda_{2}^{\ell}\right) & \Sigma^{u}\left(-\lambda_{2}^{\ell}\right) \\
\Sigma^{v}\left(\lambda_{1}^{\ell}\right) & \Sigma^{v}\left(-\lambda_{1}^{\ell}\right) & \Sigma^{v}\left(\lambda_{2}^{\ell}\right) & \Sigma^{v}\left(-\lambda_{2}^{\ell}\right)
\end{array}\right]\left\{\begin{array}{c}
A^{\ell} r^{\lambda_{1}^{\ell}} \\
B^{\ell} r^{-\lambda_{1}^{\ell}} \\
C^{\ell} r_{2}^{\lambda_{2}^{\ell}} \\
D^{\ell} r^{-\lambda_{2}^{\ell}}
\end{array}\right\}
$$

Moreover, introducing $r=a_{k}$ and $r=b_{k}$ to be the radii of boundaries of the lamina $k$, yields:

$$
\left\{\frac{U^{\ell}\left(a_{k}\right)}{\cos \alpha_{\ell} \theta} \quad \frac{U^{\ell}\left(b_{k}\right)}{\cos \alpha_{\ell} \theta} \quad \frac{V^{\ell}\left(a_{k}\right)}{\sin \alpha_{\ell} \theta} \quad \frac{V^{\ell}\left(b_{k}\right)}{\sin \alpha_{\ell} \theta}\right\}^{\mathrm{T}}=\boldsymbol{T}\left\{\begin{array}{llll}
A^{\ell} & B^{\ell} & C^{\ell} & D^{\ell}
\end{array}\right\}^{\mathrm{T}}
$$

where $\boldsymbol{T}$ is a square matrix following from the comparison of (17) and (16). The unknown integration constants are then expressed by the displacements on the boundaries of the $k$-th lamina. Substituting them back to (17) gives distribution of $U^{\ell}, V^{\ell}$ in the lamina $k$ in terms of the boundary displacements.

The last step in the computation is expressing the strains and stresses in each lamina. It is enough to use (17) and substitute them in the expression (2) to obtain strains and to (3) to get stresses.

\section{OVERALL RESPONSE OF THE LAMINATED STRUCTURE IN THE PLAIN STRAIN STATE}

In order to introduce interfacial conditions we try to express the above formulas in more compact way. Since our aim is to separate the series for distinctive laminas $(k)$ to individual members $(\ell)$, for which equivalence between expressions for given $\mathrm{k}$ and $\ell$ can be defined. For this reason, identities (14), (15), and (17) are recorded in a comprehensive form as:

$$
\left\{\begin{array}{l}
P_{a}^{\ell}(k) \\
P_{b}^{\ell}(k) \\
S_{a}^{\ell}(k) \\
S_{a}^{\ell}(k)
\end{array}\right\}=\boldsymbol{K}^{\ell}(k, \omega)\left\{\begin{array}{c}
U_{a}^{\ell}(k) \\
U_{b}^{\ell}(k) \\
V_{a}^{\ell}(k) \\
V_{a}^{\ell}(k)
\end{array}\right\}
$$

where:

$$
P_{a}^{\ell}=2 \pi \sigma_{r}^{\ell} a_{k}, P_{b}^{\ell}=2 \pi \sigma_{r}^{\ell} b_{k}, S_{a}^{\ell}=2 \pi \sigma_{r \theta}^{\ell} a_{k}, S_{a}^{\ell}=2 \pi \sigma_{r \theta}^{\ell} b_{k},
$$

$\boldsymbol{K}^{\ell}$ is a square symmetric matrix relating tractions and displacements along interfaces $r=a_{k}$, and $r=b_{k}$ inside the lamina $k$. For the whole structure it has to hold on the interface between the layer $k$ and $k-1$ :

$$
\begin{aligned}
& r=a_{k}=b_{k-1}, \\
& P_{a}^{\ell}(k)=P_{b}^{\ell}(k-1)=P_{k}^{\ell}, S_{a}^{\ell}(k)=S_{b}^{\ell}(k-1)=S_{k}^{\ell}, U_{a}^{\ell}(k)=U_{b}^{\ell}(k-1) V_{a}^{\ell}(k)=V_{b}^{\ell}(k-1)
\end{aligned}
$$


On the boundaries, for $r=a$ and $r=b$ the force-load (external load) has to be developed in Fourier series and the coefficients in it must be in compliance with the adjacent layers. From this consideration it follows that the linear algebraic system of equations can be stored as:

$$
\boldsymbol{P}=\boldsymbol{K}(\omega) \boldsymbol{U}
$$

where the right hand side is the vector describing the interfacial radial and shear tractions, $\boldsymbol{K}$ is the stiffness matrix of the structure involving the eigenfrequency $\omega, \boldsymbol{U}$ means the vector of radial and hoop displacements on the interfaces.

Exactly in the same way the extension to the generalized plain strain can be formulated, although necessary tools have to be implied for non-linear system of equations. Since the laminas are assumed as very thin, reasonable simplifications can be applied.

\section{EIGENPARAMETERS}

The stress tensor $\boldsymbol{\sigma}$ was related with the strain tensor $\boldsymbol{\varepsilon}$ and the eigenstrain tensor $\boldsymbol{\mu}$ through the material stiffness matrix, and the supposition is applied: $L_{i j}=L_{j i}, G_{r \theta}$ are stiffness coefficients, $L_{r r}=L_{\theta \theta}, L_{r r}-L_{r \theta}=2 G_{r \theta}$. From these assumptions it follows that only two material coefficient describe each lamina.

The Hooke law can now be written as:

$$
\begin{aligned}
& \sigma_{r}=L_{r r}\left(\varepsilon_{r}-\mu_{r}\right)+L_{r \theta}\left(\varepsilon_{\theta}-\mu_{\theta}\right)=L_{r r}\left(\frac{\partial u}{\partial r}-\mu_{r}\right)+L_{r \theta}\left(\frac{1}{r} \frac{\partial v}{\partial \theta}+\frac{u}{r}-\mu_{\theta}\right) \\
& \sigma_{\theta}=L_{r \theta}\left(\varepsilon_{r}-\mu_{r}\right)+L_{\theta \theta}\left(\varepsilon_{\theta}-\mu_{\theta}\right)=L_{r \theta}\left(\frac{\partial u}{\partial r}-\mu_{r}\right)+L_{\theta \theta}\left(\frac{1}{r} \frac{\partial v}{\partial \theta}+\frac{u}{r}-\mu_{\theta}\right) \\
& \sigma_{r \theta}=G_{r \theta}\left(\varepsilon_{r \theta}-\mu_{r \theta}\right)=G_{r \theta}\left(\frac{1}{r} \frac{\partial u}{\partial \theta}+\frac{\partial v}{\partial r}-\frac{v}{r}-\mu_{r \theta}\right)
\end{aligned}
$$

Similarly to the displacements the eigenstrains will be expanded into series with the $\ell$ th components:

$$
\mu_{r}^{\ell}(r, \theta)=R^{\ell}(r) \cos \alpha \theta, \mu_{\theta}^{\ell}(r, \theta)=F^{\ell}(r) \cos \alpha \theta, \mu_{r \theta}^{\ell}(r, \theta)=M^{\ell}(r) \sin \alpha \theta
$$

Components of strains and stresses are then expressed as (identification $\ell$ of the number in the series is dropped out and the prime denotes derivative by $r$ ), in what follows only one term of Fourier's series will be considered, i.e. $\ell=$ const.:

$$
\varepsilon_{r}=U^{\prime} \cos \alpha \theta, \quad \varepsilon_{\theta}=\frac{1}{r}(\alpha V+U) \cos \alpha \theta, \quad \varepsilon_{r \theta}=\left(\frac{-\alpha}{r} U+V^{\prime}-\frac{1}{r} V\right) \sin \alpha \theta
$$




$$
\begin{gathered}
\sigma_{r}=\left[L_{r r}\left(U^{\prime}-R\right)+\frac{L_{r \theta}}{r}(\alpha V+U-F)\right] \cos \alpha \theta, \quad \sigma_{\theta}=\left[L_{r \theta}\left(U^{\prime}-R\right)+\frac{L_{\theta \theta}}{r}(\alpha V+U-F)\right] \cos \alpha \theta \\
\sigma_{r \theta}=G_{r \theta}\left(\frac{-\alpha}{r} U+V^{\prime}-\frac{1}{r} V-M\right) \sin \alpha \theta
\end{gathered}
$$

The first equation of equilibrium is now expressed in terms of amplitudes of displacements and eigenstrains as:

$$
U^{\prime \prime}+\frac{U^{\prime}}{r}+A_{1} \frac{U}{r^{2}}+A_{2} \frac{V^{\prime}}{r}+A_{3} \frac{V}{r^{2}}=R^{\prime}+\left(\Lambda F^{\prime}+\Phi_{1} M+\Psi_{1} R\right) \frac{1}{r}-F \frac{1}{r^{2}}
$$

where

$$
\begin{aligned}
& A_{1}=\frac{-\left(L_{r r}+\alpha^{2} G_{r \theta}\right)}{L_{r r}}, \quad A_{2}=\frac{\alpha\left(L_{r \theta}+G_{r \theta}\right)}{L_{r r}}, \quad A_{3}=\frac{-\alpha\left(L_{r r}+G_{r \theta}\right)}{L_{r r}}, \quad \Lambda=\frac{L_{r \theta}}{L_{r r}}, \\
& \Phi_{1}=\frac{\alpha G_{r \theta}}{L_{r r}}, \quad \Psi_{1}=\frac{2 G_{r \theta}}{L_{r r}}
\end{aligned}
$$

The second equation of equilibrium involving the eigenstrains are:

$$
V^{\prime \prime}+\frac{V^{\prime}}{r}+B_{1} \frac{V}{r^{2}}+B_{2} \frac{U^{\prime}}{r}+B_{3} \frac{U}{r^{2}}=M^{\prime}+\left(2 M+\Psi_{2} R\right) \frac{1}{r}+\Phi_{2} \frac{F}{r^{2}}
$$

where

$$
B_{1}=\frac{-\left(G_{r \theta}+\alpha^{2} L_{r r}\right)}{G_{r \theta}}, B_{2}=\frac{-\alpha\left(L_{r \theta}+G_{r \theta}\right)}{G_{r \theta}}, B_{3}=\frac{-\alpha\left(L_{r r}+G_{r \theta}\right)}{G_{r \theta}}, \Psi_{2}=-\frac{\alpha L_{r \theta}}{G_{r \theta}}, \Phi_{2}=-\frac{\alpha L_{r r}}{G_{r \theta}}
$$

Strain in radial direction:

$$
\begin{aligned}
& \varepsilon_{r}=\left\{\lambda_{1} \alpha\left[\left(L_{r r}+G_{r \theta}\right)-\lambda_{1}\left(L_{r \theta}+G_{r \theta}\right)\right] A r^{\lambda_{1}-1}-\lambda_{1} \alpha\left[\left(L_{r r}+G_{r \theta}\right)+\lambda_{1}\left(L_{r \theta}+G_{r \theta}\right)\right] B r^{-\lambda_{1}-1}+\right. \\
& +\lambda_{2} \alpha\left[\left(L_{r r}+G_{r \theta}\right)-\lambda_{2}\left(L_{r \theta}+G_{r \theta}\right)\right] C r^{\lambda_{2}-1}-\lambda_{2} \alpha\left[\left(L_{r r}+G_{r \theta}\right)+\lambda_{2}\left(L_{r \theta}+G_{r \theta}\right)\right] D r^{-\lambda_{2}-1}+ \\
& \left.+\frac{4 \alpha^{2}\left(L_{r r}-G_{r \theta}\right)}{(4 \alpha-1) L_{r r}} R+\frac{\alpha\left(\alpha^{2} L_{r r}-4 G_{r \theta}\right)}{(4 \alpha-1) L_{r r}} M\right\} \cos \alpha \theta
\end{aligned}
$$

Hoop strain is derived as: 


$$
\begin{aligned}
& \varepsilon_{\theta}=\frac{1}{r} \alpha\left\{\left[\lambda_{1}^{2} L_{r r}-\left(L_{r r}+\alpha^{2} G_{r \theta}\right)+\left(L_{r r}+G_{r \theta}\right)-\lambda_{1}\left(L_{r \theta}+G_{r \theta}\right)\right] A r^{\lambda_{1}}+\right. \\
& +\left[\lambda_{1}^{2} L_{r r}-\left(L_{r r}+\alpha^{2} G_{r \theta}\right)+\left(L_{r r}+G_{r \theta}\right)+\lambda_{1}\left(L_{r \theta}+G_{r \theta}\right)\right] B r^{-\lambda_{1}}+ \\
& +\left[\lambda_{2}^{2} L_{r r}-\left(L_{r r}+\alpha^{2} G_{r \theta}\right)+\left(L_{r r}+G_{r \theta}\right)-\lambda_{2}\left(L_{r \theta}+G_{r \theta}\right)\right] C r^{\lambda_{2}}+ \\
& +\left[\lambda_{2}^{2} L_{r r}-\left(L_{r r}+\alpha^{2} G_{r \theta}\right)+\left(L_{r r}+G_{r \theta}\right)+\lambda_{2}\left(L_{r \theta}+G_{r \theta}\right)\right] D r^{-\lambda_{2}}+ \\
& \left.+\frac{\left(\alpha^{2}+1\right)}{\alpha\left(\alpha^{2}-1\right)} F+\frac{\alpha^{3}\left(2 G_{r \theta}-L_{r r}\right)-4 \alpha+4 \alpha\left(L_{r r}-G_{r \theta}\right)}{(4 \alpha-1) L_{r r}} R r+\frac{\alpha^{2}\left(2 G_{r \theta}-L_{r r}\right)-4 G_{r \theta}}{(4 \alpha-1) L_{r r}} M r\right\} \cos \alpha \theta
\end{aligned}
$$

Shear strain is:

$$
\begin{aligned}
& \varepsilon_{r \theta}=\frac{1}{r}\left\{-\alpha^{2}\left[\left(L_{r r}+G_{r \theta}\right)-\lambda_{1}\left(L_{r \theta}+G_{r \theta}\right)\right]+\lambda_{1}\left[\lambda_{1}^{2} L_{r r}-\left(L_{r r}+\alpha^{2} G_{r \theta}\right)\right]-\left[\lambda_{1}^{2} L_{r r}-\left(L_{r r}+\alpha^{2} G_{r \theta}\right)\right] A r^{\lambda_{1}}+\right. \\
& +\left[-\alpha^{2}\left[\left(L_{r r}+G_{r \theta}\right)+\lambda_{1}\left(L_{r \theta}+G_{r \theta}\right)\right]-\lambda_{1}\left[\lambda_{1}^{2} L_{r r}-\left(L_{r r}+\alpha^{2} G_{r \theta}\right)\right]-\left[\lambda_{1}^{2} L_{r r}-\left(L_{r r}+\alpha^{2} G_{r \theta}\right)\right] B r^{-\lambda_{1}}+\right. \\
& +\left[-\alpha^{2}\left[\left(L_{r r}+G_{r \theta}\right)-\lambda_{2}\left(L_{r \theta}+G_{r \theta}\right)\right]+\lambda_{2}\left[\lambda_{2}^{2} L_{r r}-\left(L_{r r}+\alpha^{2} G_{r \theta}\right)\right]-\left[\lambda_{2}^{2} L_{r r}-\left(L_{r r}+\alpha^{2} G_{r \theta}\right)\right]\right] r^{\lambda_{2}}+ \\
& +\left[-\alpha^{2}\left[\left(L_{r r}+G_{r \theta}\right)+\lambda_{2}\left(L_{r \theta}+G_{r \theta}\right)\right]-\lambda_{2}\left[\lambda_{2}^{2} L_{r r}-\left(L_{r r}+\alpha^{2} G_{r \theta}\right)\right]-\left[\lambda_{2}^{2} L_{r r}-\left(L_{r r}+\alpha^{2} G_{r \theta}\right)\right] D r^{-\lambda_{2}}+\right. \\
& \left.-\frac{2 \alpha}{\alpha^{2}-1} F-\frac{4 \alpha^{3}\left(L_{r r}-G_{r \theta}\right)}{(4 \alpha-1) L_{r r}} R r-\frac{\alpha^{2}\left(\alpha^{2} L_{r r}-4 G_{r \theta}\right)}{(4 \alpha-1) L_{r r}} M r\right\} \sin \alpha \theta
\end{aligned}
$$

In order to introduce interfacial conditions we try to express the above formulas in more compact way. Our aim is to separate the series for distinctive laminas $(k)$ to individual members $(\ell)$, for which equivalence between expressions for given $k$ and $\ell$ can be defined. For this reason, identities (20), (22), and (19) are recorded in comprehensive form as:

$$
\left\{\begin{array}{l}
P_{a}^{\ell}(k) \\
P_{b}^{\ell}(k) \\
S_{a}^{\ell}(k) \\
S_{a}^{\ell}(k)
\end{array}\right\}=\boldsymbol{K}^{\ell}(k)\left\{\begin{array}{l}
U_{a}^{\ell}(k) \\
U_{b}^{\ell}(k) \\
V_{a}^{\ell}(k) \\
V_{a}^{\ell}(k)
\end{array}\right\}+\boldsymbol{Q}^{\ell}(k)\left\{\begin{array}{c}
R^{\ell}(k) \\
F^{\ell}(k) \\
M^{\ell}(k)
\end{array}\right\}
$$

where $\boldsymbol{K}^{\ell}$ is a square matrix relating tractions and displacements along interfaces $r=a_{k}$, and $r=b_{k}, Q^{\ell}$ is the matrix ( $\left.3 \times 4\right)$ relating the same tractions and eigenstrains inside the lamina $k$. On the boundaries, for $r=a$ and $r=b$ the force-load (external load) has to be developed in Fourier series and the coefficients in it must be in compliance with the adjacent layers. From this consideration it follows that the linear algebraic system of equations can be stored as:

$$
\boldsymbol{P}=\boldsymbol{K} \boldsymbol{U}+\boldsymbol{Q \mu}
$$

where the right hand side is the vector describing the interfacial radial and shear tractions, $\boldsymbol{K}$ is the stiffness matrix of the structure, $\boldsymbol{U}$ means the vector of radial and hoop 
displacements on the interfaces, $\boldsymbol{Q}$ is the matrix of eigenstrain influences and eventually, $\boldsymbol{\mu}$ is the vector of all eigenstrains in layer, which are in the layers uniform.

\section{OPTIMAL PRESTRESS OF LAMINATED ARCHES}

A thick-walled composite arch consisting of many different cylindrically orthotropic layers is loaded by arbitrary loading, preferably by surface tractions and by piecewise uniform in radial direction eigenstrains in selected layers. A theoretical framework is established for evaluation of internal stress fields. An optimization procedure is implemented to find eigenstrain distributions that adjust the eigenfrequencies and stresses in the layers to selected levels, while allowing the application of certain ranges of fiber prestrain magnitude to reduce fiber waviness. A particular fabrication process that utilizes fiber prestress as a source of the layer eigenstrains is analyzed, and a distribution of the fiber prestress forces is found that produces a desired distribution of the hoop and radial stresses in the arch wall. "Almost rigid" mandrel is used and an "almost rigid" box, the layers in which are created, is prepared for fabrication of the composite. Both mandrel and box stiffnesses and the chosen prestress magnitude in the first layer influence the distribution. It is shown that application of a high fiber prestress that is constant through the wall thickness can be harmful as it may generate very high hoop stress gradients.

One of the potential applications to composite materials is in structures subjected primarily to compressive loads, such as submersibles, pilot cockpits, aircrafts, etc. An incentive is the relatively high compressive strength of carefully fabricated thick samples, e.g. $1,140 \mathrm{MPa}(209 \mathrm{ksi})$ in an AS4/3501-6 carbon/epoxy system; even the more frequently reported magnitude of about $700 \mathrm{MPa}$ for this system is attractive. Cylindrical, elliptic, or spherical shapes are typically preferred in such applications, and if the wall to diameter ratios is small, then, regardless of absolute size, the structure responds to external compression as a thin-walled cylinder or sphere, with a nearly uniform distribution of load-induced stresses through the wall thickness. However, significant residual stress gradients can be caused by fabrication and processing, e.g. by fiber prestresst hat may be needed to reduce fiber waviness, or by non-uniform cooling from the curing temperature. In superposition with external loads, the non-uniform residual stresses may lead to premature failure. The purpose of this chapter is to establish a theoretical framework for evaluation of optimal internal eigenstrain fields that generate prescribed stress distribution in laminated composite arches loaded by radial external pressure. Any number of different cylindrically orthotropic layers can be considered. It is shown that a high fiber prestress that is applied uniformly through the wall thickness may cause very unfavorable residual stresses. However a variable prestress can be identified to provide an optimal stress distribution in the structure. This chapter utilizes the theory outlined in previous sections. An application to the design of a stressed arch structure by one sine wave is presented.

Under the assumption of plane strain or plane stress in the width direction, by introducing stress components Hamilton's principle is applied to derive the equations of dynamic equilibrium and natural boundary conditions of an arch. In order to treat vibration and also possibel stability problems of an arch subjected to initial axial stress $\lambda_{\theta}$, i.e. in hoop 
direction additional work due to this stress which is assumed either to remain unchanged during vibrating and/or buckling or changing according to the displacement change during time dependent process is taken into consideration. Here only the first part is taken as the starting supposition.

The principle for the present problems may be expressed for an arbitrary time interval $t_{1}$ to $t_{2}$ as follows:

$$
\begin{aligned}
I(\lambda)= & \int_{t_{1}}^{t_{2}} \int_{\Omega}\left\{\left(\sigma_{r} \delta \varepsilon_{r}+\sigma_{\theta} \delta \varepsilon_{\theta}+\sigma_{z} \delta \varepsilon_{z}+2\left(\tau_{r \theta} \delta \varepsilon_{r \theta}+\tau_{r z} \delta \varepsilon_{r z}+\tau_{\theta z} \delta \varepsilon_{\theta z}\right)+\lambda_{\theta} \sigma_{\theta} \delta \varepsilon_{\theta}+\lambda_{z} \sigma_{z} \delta \varepsilon_{z}-\right.\right. \\
& \left.\rho\left(\frac{\partial u}{\partial t} \delta \frac{\partial u}{\partial t}+\frac{\partial v}{\partial t} \delta \frac{\partial v}{\partial t}+\frac{\partial w}{\partial t} \delta \frac{\partial w}{\partial t}\right)\right\} \mathrm{d} \Omega \mathrm{d} t=0
\end{aligned}
$$

where the symbol $\delta$ indicates the variation (special Gateau's derivative), $\rho$ denotes the mass density. The above Hamilton's variaitonal principle is written in general form, assumptions introduced in previous chapters have to be introduced.

Our aim now is to determine the eigenstrains in such a way that the selected stresses will be optimally distributed though the structure. In other words, we require (for simplicity only hoop and axial eigenstrains are selected to be the design parameters, as they are the only reasonable directions of prestressing). For that (41) serve a tool on how to calculate the relation between stresses and eigenstresses, and afterwards also the relation between displacements and eigenstresses. This relation is then used in the variational principle (42).

First the statement that the prestresses cannot exceed a reasonable limit to avoid possible waveness or kinking. This condition leads us to the following condition:

$$
I\left(\lambda_{\theta}^{j}, \lambda_{z}^{j}\right)=\sum_{k}\left(P_{k}-\sum_{j}\left(G_{k j}^{\theta} \lambda_{\theta}^{j}+G_{k j}^{z} \lambda_{z}^{j}\right)^{2} \rightarrow\right. \text { minimum }
$$

where $\boldsymbol{G}^{\theta}$ is the influence matrix for eigenstresses $\lambda_{\theta}^{j}$ and $\boldsymbol{G}^{z}$ is the influence matrix for eigenstresses $\lambda_{z}^{j}$. Vector $\boldsymbol{P}$ in (43) is the representative of an arbitrary selected external load, as eigenfrequences are to be optimized. The problem (43) results in interesting facts, such as that the number of eigenparameters involved in the optimization is equal to two times number of laminas minus one (eigenparameters are introduced in both directions, $\theta$ and $z$, and if this condition is fulfilled the distribution of stresses through the thickness is uniform for arbitraryly selected external load satisfying the conditions of admissibility, as, for instance, the required symmetry. These assertions are proved in [9] for symmetric cylinder and in [10] the proofs are extended to arches. This is true also vice versa.

Second, assuming constant values of eigenparameters inside of each lamina (but different mutually), from (42) it follows that

$$
\boldsymbol{K}(\lambda) \boldsymbol{u}+\omega \boldsymbol{M u}=\mathbf{0}
$$

where $\boldsymbol{K}$ is the stiffness matrix of the structure and $\boldsymbol{M}$ is the mass matrix. Note that the simplification concerning constant eigenparameters in each lamina can be generalized as quite general formulas have been derived in previous chapters. 
Differentiating (44) by $\lambda_{i}$, where $\lambda_{i}$ stands for either $\lambda_{\theta}^{j}$ or $\lambda_{z}^{j}$, and accepting the requirement for extreme eigenfrequency, i.e. $\frac{\partial \omega}{\partial \lambda_{i}}=0$ for every admissible $i$, condition (43) is obtained for $P_{k}=0$. Ones the eigenparameters are determined form (43) (autonomic system is obtained and the value of one of the eigenparameters has to be selected and given), the equations (44) then provide us with the optimal eigenfrequency.

\section{EXAMPLE}

The arch has the following dimensions and material properties: the length $1=1 \mathrm{~m}$, the external radius $\mathrm{r}=2 \mathrm{~m}$, the thickness $=5 \mathrm{~mm}, \beta=0.5 \mathrm{rad}, E=208 \times 10^{9} \mathrm{~N} / \mathrm{m} 2, \rho=7833$ $\mathrm{kg} / \mathrm{m} 3, v=0.29$.

Five equidistant layers are considered through the thickness of the arch. A special layer is positioned in only one inner layer with $E=200 \times 10^{8} \mathrm{~N} / \mathrm{m} 2$ and will move for successive examples from the layer one to five. The natural frequencies are calculated for these particular cases. They are displayed in Fig. 2 for results without eigenparameters (prestress). Number one belongs to the position of the weaker material in layer one, etc., the numbering is from the outer boundary to the lower one.

In Fig. 3 the results from the current approach with the same geometry and material properties are depicted. The optimal eigenparameters and stresses are seen from the next picture, Fig. 4.

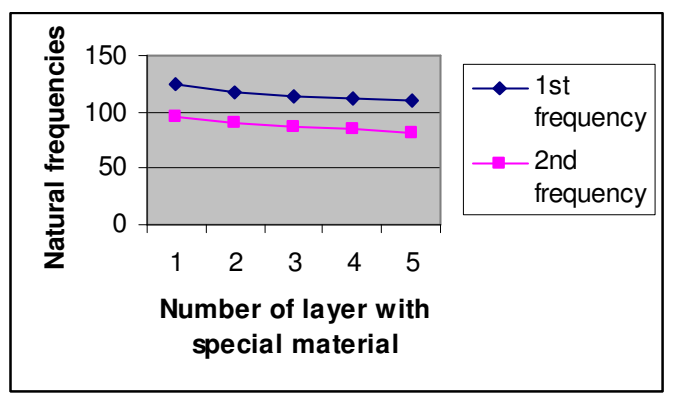

Figure 2. Eigenfrequencies without prestressing

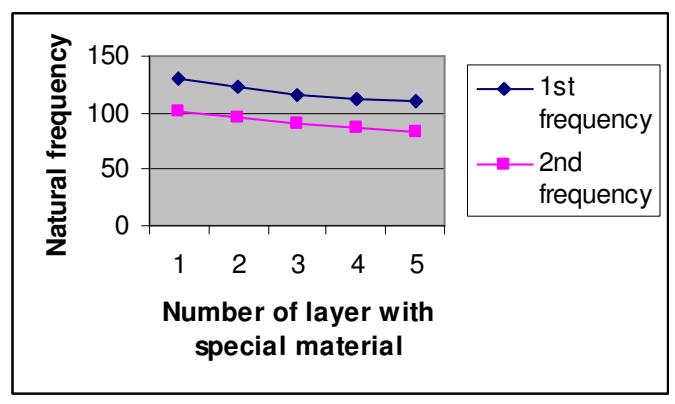

Figure 3. Eigenfrequencies with optimal prestressing 

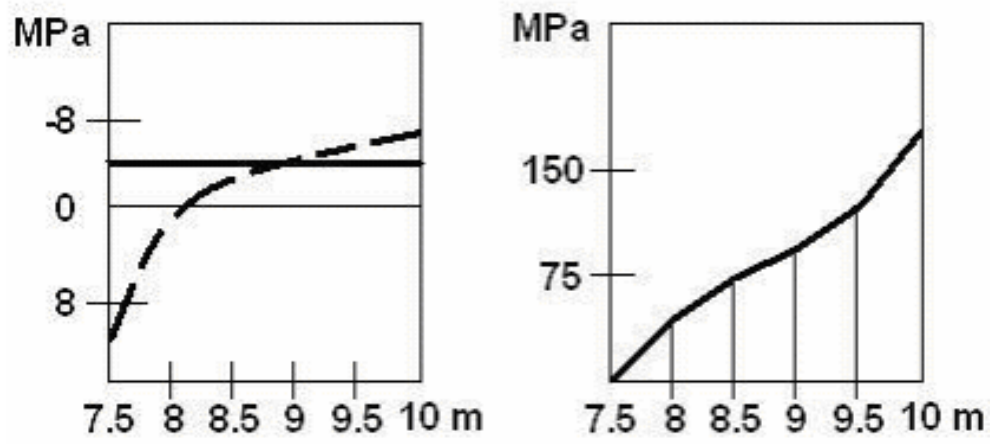

- - - stress without eigenstrain

optimal stress

Figure 4. Amplitudes of stress distribution in the length of the arch and optimal distribution of prestress

The results show that the eigenfrequencies heighten in optimized case. This fact is noticeable for weaker lamina at the lower position. If the weaker lamina is introduced at the outer position the differences between optimized and not optimized cases are not demonstrable.

\section{CONCLUSIONS}

In this paper new procedure for calculating optimal (a posteriori) prestress of laminated composite arch is suggested. The solution is presented in cylindrical coordinates and is formulated in pseudo-three dimensions. Semi-analytical solution is applied and waves in time and hoop direction separate the arguments of constitutive quantities into radial direction in which ordinary differential equations are to be solved. For isotropic case quite simple solution is obtained and very fast algorithm for computer can be created.

A series of examples of application of the presented approach consists in simplifying the problem to two dimensions. In the examples one weaker layer changes its position in the laminated composite. Two first natural (eigen) frequencies are compared: the results from the dynamics of structure without prestress and that from the current approach, in which all laminas are optimally prestressed but one prestress is selected according to requirement of excluding possible tension in laminas, for example. In case of weaker layers being located at the inner boundary the results are relatively improved by the prestress but if the weaker lamina is located at the outer boundary of the laminated composite structure almost the same results for optimally prestressed and without prestress is attained.

\section{ACKNOWLEDGEMENTS}

This work has been financially supported by GAČR, project number P105/10/0266. 


\section{REFERENCES}

[1] Kochupillai J., Ganesan N., Padmanabhan C. "A semi-analytical coupled finite element formulation for composite shells conveying fluid". Journal of Sound and Vibration 258, 2, 287-307, 2002

[2] Ramasamy S., Ganesan N., "Vibration and damping analysis of fluid filled orthotropic cylindrical shells with constrained viscoelastic damping". Computers and Structures 70 , 363-376, 1999.

[3] Patel B.P., Ganapathi M., Makhecha D.P., "Hygrothermal effects on the structural behaviour of thick composite laminates using higher-order theory". Composite Structures $56,1,25-34,2002$

[4] Pi Y.L., Trahair N.S., "Three-dimensional nonlinear analysis of elastic arches". Engineering Structures 18, 1, 49-63, 1996.

[5] Pi Y.L., Bradford M.A., "Elastic flexural-torsional buckling of continuously restrained arches". Int. J. Solids and Structures 39, 8, 2299-2322, 2002.

[6] Lim N.H., Kang Y.J., "Out of plane stability of circular arches”. Int. J. Mech. Sci. 46, 8, 1115-1137, 2004.

[7] Prochazka P.P., Dolezel V., Lok T.-S., "Optimal shape design for minimum Lagrangian”. Eng. Anal. with Bound. Elem. 33, 447-455, 2009.

[8] Hiroyuki M., "Free vibration and stability of laminated composite circular arches subjected to initial axial stress“. Journal of Sound and Vibration 271, 651-670, 2004

[9] Prochazka P., "Deterministic and stochastic optimization of composite cylindrical laminates”. Int. J. Solids and Structures 40, 7109-7127, 2003

[10] Yakoumi A., "Selected applications of eigenparameter theory to laminated structures". Doctoral Thesis CTU in Prague, March 2008 